\title{
Catalogue of Neotropical Curtonotidae (Diptera, Ephydroidea)
}

\author{
Ramon Luciano Mello ${ }^{1}$ \& Alessandre Pereira-Colavite ${ }^{2}$ \\ ${ }^{1}$ Universidade Federal de Mato Grosso do Sul (UFMS), Instituto de Biociências (INBI0), Laboratório de Sistemática de Diptera (LSD). \\ Campo Grande, MS, Brasil. ORCID: 0000-0002-1914-5766. E-mail: ramon.mello@ufms.br \\ 2 Universidade Federal da Paraíba (UFPB), Centro de Ciências Exatas e da Natureza (CCEN), Departamento de Sistemática e Ecologia (DSE). \\ João Pessoa, PB, Brasil. ORCID: 0000-0002-7660-8384. E-mail: alepercol@gmail.com
}

\begin{abstract}
The Neotropical species of Curtonotidae are updated and catalogued. A total of 33 species names are listed, including two fossil taxa and one nomem dubium. Valid and invalid names and synonyms are presented, totaling 45 names. Bibliographic references are given to all listed species, including information about name, author, year of publication, page number, type species and type locality. Lectotype and paralectotypes are designated to Curtonotum punctithorax (Fischer, 1933). Curtonotum simplex Schiner, 1868 stat. rev. is recognized as a valid name.
\end{abstract}

Key-Words. Acalyptratae; Curtonotum; Hunchbacked flies; Lectotype; Paralectotype; Schizophora; Type material.

\section{INTRODUCTION}

Curtonotidae, also called hunchbacked flies or quasimodo flies, is a small family of dipterous Acalyptratae with worldwide distribution. Although the family might be found in all biogeographic regions, they occur mainly in the tropical and subtropical areas, from sea level to high altitudes $(+2,000 \mathrm{~m})$ (Kirk-Spriggs, 2010a). Four living genera are known: Axinota Wulp (12 spp.) occurring in Afrotropical, Oriental and Australasian/ Oceanic regions; Curtonotum Macquart (76 spp.) occurring in every zoogeographic region except the Australasian/Oceanic region; Cyrtona s.l. Séguy (4 spp.) and Tigrisomyia Kirk-Spriggs (4 spp.), both known only to Afrotropical region so far (KirkSpriggs, 2010a, 2010b, 2011; Klymko \& Marshall, 2011; Kirk-Spriggs \& Wiegmann, 2013). Fossil species are known from the Miocene Dominican amber to Curtonotum (1 sp.) and Depressonotum Grimaldi \& Kirk-Spriggs (1 sp.) (Grimaldi \& Kirk-Spriggs, 2012).

The entire fauna of the New World region includes only species in Curtonotum, currently accepted as a probably paraphyletic group in a sensu lato form. Nevertheless, all Neotropical species form a monophyletic clade, being commonly referred to as Curtonotum sensu stricto and characterized by elongate spermathecal ducts (Klymko \& Marshall, 2011). The Neotropical species can be distinguished by the following characteristics: (1) small to medium-sized (length 4-12 mm) and distinctively hunchbacked flies; (2) coloring usually grayish to yellow, often with spots, stripes, or speckles on thorax and pigment patterns on abdomen; (3) arista plumose, with long dorsal and ventral rays; (4) wing pigmentation varying from hyaline to lightly fumose or boldly patterned; (5) subcostal vein complete, with cell cup present and cells $\mathrm{dm}$ and bm confluent; (6) costal vein with humeral and subcostal breaks; and (7) with several spinelike bristles between apices of $R_{1}$ and $R_{2+3}$ veins (Marshall et al., 2010).

The biology is scarcely known for curtonotids. The Afrotropical Curtonotum saheliense Tsacas, 1977 and the Palaearctic C. simile Tsacas, 1977 are saprophagous on damaged grasshopper and locust egg pods rather than being truly parasitic (Greathead, 1958; Kirk-Spriggs, 2008). The Nearctic Curtonotum helvum (Loew, 1862) occurs in sand dunes and the larvae probably develop on the same substrate as it's congenial of the Old World (Meier et al., 1997; Marshall et al., 2010; Marshall, 2012). Adult Neotropical species occurs on dung baits and in association with tree falls (Marshall et al., 2010; Klymko \& Marshall, 2011), but oviposition has not been observed and larval habits remain unknown.

The present catalogue covers all the names related to the Neotropical fauna of Curtonotidae. We list here a total of 45 names, including 33 valid species (including two fossils and one nomem dubium) and two taxonomically valid genera. A total of 44 references are given. According with the Article 74.1, of the $4^{\text {th }}$ edition of the International Code of Zoological Nomenclature (ICNZ, 1999), the lectotype and paralectotypes of Curtonotum punctithorax (Fischer, 1933) are here designated in order to fix the identity of the name (Fig. 1). Curtonotum simplex Schiner, 1868 (Fig. 2), stat. rev. is removed from incertae sedis and placed as a valid name. 

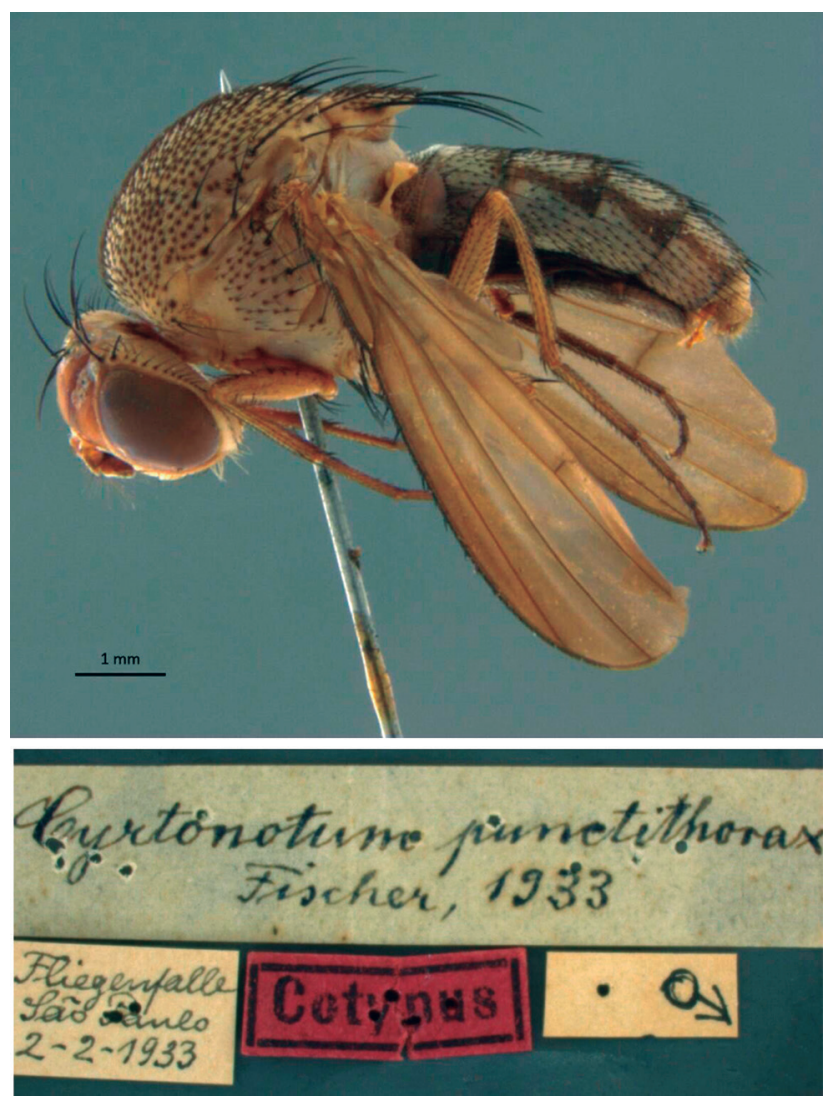

Figure 1. Curtonotum punctithorax (Fischer, 1933), lectotype male herein designated. (A) Habitus, lateral view; (B) Labels. Images provided by the Museu de Zoologia, Universidade de São Paulo.

\section{MATERIAL AND METHODS}

The information presented in this catalogue is focused in species level, since Curtonotidae and Curtonotum occur in other biogeographic regions. Species are presented, in alphabetical order of specific name, with the following data: name, author, year of publication, page number, type locality, distribution, references, and sex of the primary type, including its depository institution. Taxonomically valid names are listed in bold, and synonyms and other invalid spellings are listed in italics, below the reference to the corresponding name.

Acronyms used in the catalogue are: $\mathbf{A M N H}$ : American Museum of Natural History, New York, USA; CBFC: Museo Nacional de Historia Natural, La Paz, Bolivia; DEBU: University of Guelph Insect Collection, Guelph, Canada; HNHM: Hungarian Natural History Museum, Budapest, Hungary; IBSP: Instituto Biológico de São Paulo, São Paulo, Brazil; MSNM: Museo Civico di Storia Naturale, Milan, Italy; MZUSP: Museu de Zoologia da Universidade de São Paulo, São Paulo, Brazil; NHM: The Natural History Museum, London, United Kingdom; NHMW: Naturhistorisches Museum Wien, Vienna, Austria; QCAZ: Departamento de Biologia, Pontífica Universidad Católica del Ecuador, Quito, Ecuador; SMNS: Staatliches Museum für Naturkunde Stuttgart, Stuttgart, Germany; SMTD: Staatliches Museum für Tierkunde Dresden, Dresden, Germany; USNM: National Museum of Natural History,
Washington D.C., USA; ZMUC: Zoological Museum, University of Copenhagen, Copenhagen, Denmark.

The following abbreviations and symbols are used in the catalogue: cat. $=$ catalogue; $\mathrm{ch}$. $=$ checklist; comb. $=$ combination; desc. $=$ description; design. $=$ designation; distr. $=$ distribution; lect. = lectotype; missp. = misspelling; mon. = by monotypy; $\mathrm{n}$. = new; nam. = name; occ. = occurrence; phyl. = phylogenetic; pl. = plate; reds. = redescription; Ref(s). = reference(s); rels. = relationships; syn. $=$ synonym; sync. $=$ synonymic; tax. $=$ taxonomic $; \dagger=$ fossil taxon.

\section{RESULTS}

\section{Curtonotidae Duda, 1934}

Curtonotidae Duda, 1934: 1. Type-genus Curtonotum Macquart, 1843.

Cyrtonotinae Enderlein, 1914: 326 (Nomen nudum).

\section{Curtonotum Macquart, 1843}

Curtonotum Macquart, 1843: 350. Type-species, Musca gibba Fabricius, 1805 (mon.) Junior primary homonym of Musca gibba Müller, 1776 and Musca gibba Rossi, 1794; Curtonotum taeniatum Hendel, 1913: 629 accepted as the replacement name by Thompson \& Pont (1993).

Cyrtonotum Agassiz, 1846: 108 (Unjustified emendation of Curtonotum).

Diplocentra Loew, 1862: 13 (Unjustified new name to Curtonotum).

Seliacantha Bezzi, 1895: 66 (Nomen nudum).

Selidacantha Bezzi, 1895: 66 (Nomen nudum: emendation of Seliacantha Bezzi, 1895 nomen nudum).

Parapsinota Duda, 1924: 177. (Syn.) Delfinado (1969).

adusticrus Klymko \& Marshall, 2011: 48. Type-locality: BRAZIL, Rio de Janeiro, Itatiaia. Distr.: Brazil. Refs.: Klymko \& Marshall, 2011: 11-15 (phyl. rels.), 26 (key). Holotype male (USNM).

apicale Hendel, 1913: 621. Type-locality: PERU, Junín, Chanchamayo. Distr.: Ecuador, Peru. Refs.: Hendel, 1913: 619 (key); Sturtevant, 1921: 114 (ch.), 123 (cat.); Fischer, 1933: 88 (ch.); Wirth, 1975: 1 (cat.); Klymko \& Marshall, 2011: 11-15 (phyl. rels.), 26 (key), 52 (reds.), 54 (lect. design.). Lectotype male (SMTD).

atlanticum Klymko \& Marshall, 2011: 93. Type-locality: BRAZIL, São Paulo, Campos do Jordão. Distr.: Brazil. Refs.: Klymko \& Marshall, 2011: 11-15 (phyl. rels.), 26 (key). Holotype male (MZUSP).

bathmedum Hendel, 1913: 628. Type-locality: PERU, Huanúco, Mouth of Pachieta River. Distr.: Bolivia, Peru. Refs.: Hendel, 1913: 619 (key); Sturtevant, 1921: 114 (ch.), 123 (cat.); Malloch, 1930: 325 (n. occ.); Fischer, 1933: 88 (ch.); Hennig, 1958: 528, 671 (phyl.); Wirth, 1975: 1 (cat.); Klymko \& Marshall, 2011: 11-15 (phyl. rels.), 17 (key), 43 (reds.) 44 (lect. design.). Lectotype male (SMTD). 
bathymedum Malloch, 1930: 325 (missp. of bathmedum). bivittatum Klymko \& Marshall, 2011: 39. Type-locality: ECUADOR, Napo, Jatun Sacha Res., 6 km E Misahualli. Distr.: Colombia, Costa Rica, Ecuador. Refs.: Klymko \& Marshall, 2011: 11-15 (phyl. rels.), 26 (key); Mello, 2016: 717 (cat.). Holotype male (QCAZ).

brunneum Klymko \& Marshall, 2011: 64. Type-locality: BOLIVIA, La Paz, 8 km S Chulumani, Apa Apa. Distr.: Bolivia, Peru. Refs.: Klymko \& Marshall, 2011: 11-15 (phyl. rels.), 25 (key). Holotype male (CBFC).

coriaceum Hendel, 1932: 133 (Cyrtonotum). Typelocality: BOLIVIA, Pilcomayo. Distr.: Bolivia. Refs.: Wirth, 1975: 2 (cat.); Klymko \& Marshall, 2011: 50 (tax. notes). Syntypes 2 females (SMNS).

curtispinum Klymko \& Marshall, 2011: 61 (Figs. 3, 99-105 and 212). Type-locality: BRAZIL, Rio de Janeiro, Rio de Janeiro. Distr.: Brazil. Refs.: Klymko \& Marshall, 2011: 11-15 (phyl. rels.), 25 (key). Holotype male (USNM).

decumanum Bezzi, 1914: 199 (Cyrtonotum). Typelocality: PARAGUAY, Alto Paraná, Puerto Bertoni. Distr.: Paraguay. Refs.: Lucas, 1915: 131 (notes); Sturtevant,

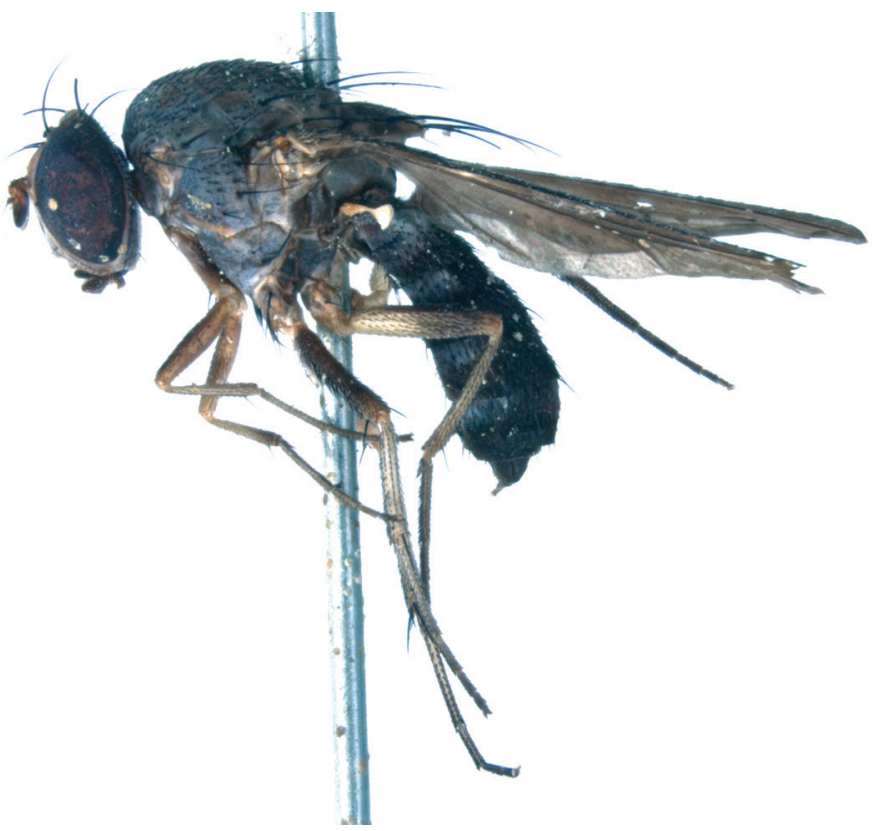

1921: 114 (ch.), 123 (cat.); Wirth, 1975: 2 (cat.); Klymko \& Marshall, 2011: 50 (tax. notes). Syntypes male and female (MSNM).

desperatum Klymko \& Marshall, 2011: 91. Type-locality: BRAZIL. Distr.: Brazil. Refs.: Klymko \& Marshall, 2011: 11-15 (phyl. rels.), 26 (key). Holotype male (NHM).

telectrodominicum Grimaldi \& Kirk-Spriggs, 2012: 3. Type-locality: DOMINICAN REPUBLIC, from amber mines in Cordillera Septentrional, northern Dominican Republic. Distr.: Dominican Republic (Miocene). Holotype female (AMNH).

flavisetum Klymko \& Marshall, 2011: 72. Type-locality: BRAZIL, Rondônia, Nova Mamoré, Vila Murtinho. Distr.: Brazil, Bolivia. Refs.: Klymko \& Marshall, 2011: 11-15 (phyl. rels.), 25 (key). Holotype male (USNM).

Comments: Although Klymko \& Marshall (2011: 75) indicated the type locality as "Mato Grosso [state], Villa Murtinho" the current location's name is "Rondônia [state], Nova Mamoré, Vila Murtinho", since Rondônia has become dismembered of part of Mato Grosso and Amazonas states in 1943, after collection of the holotype in 1922.

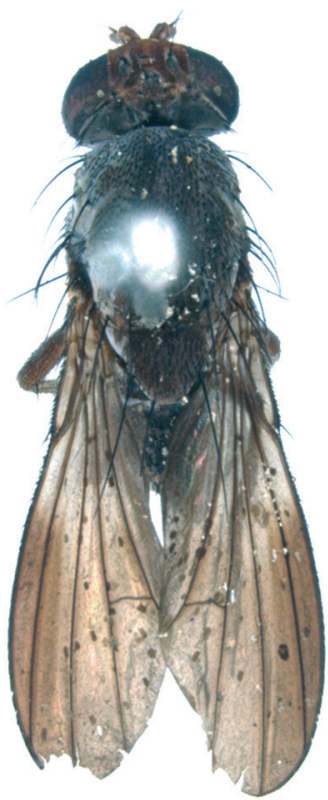

Figure 2. Curtonotum simplex Schiner, 1868, holotype female. (A) Habitus, lateral view; (B) Habitus, dorsal view; (C) Labels.
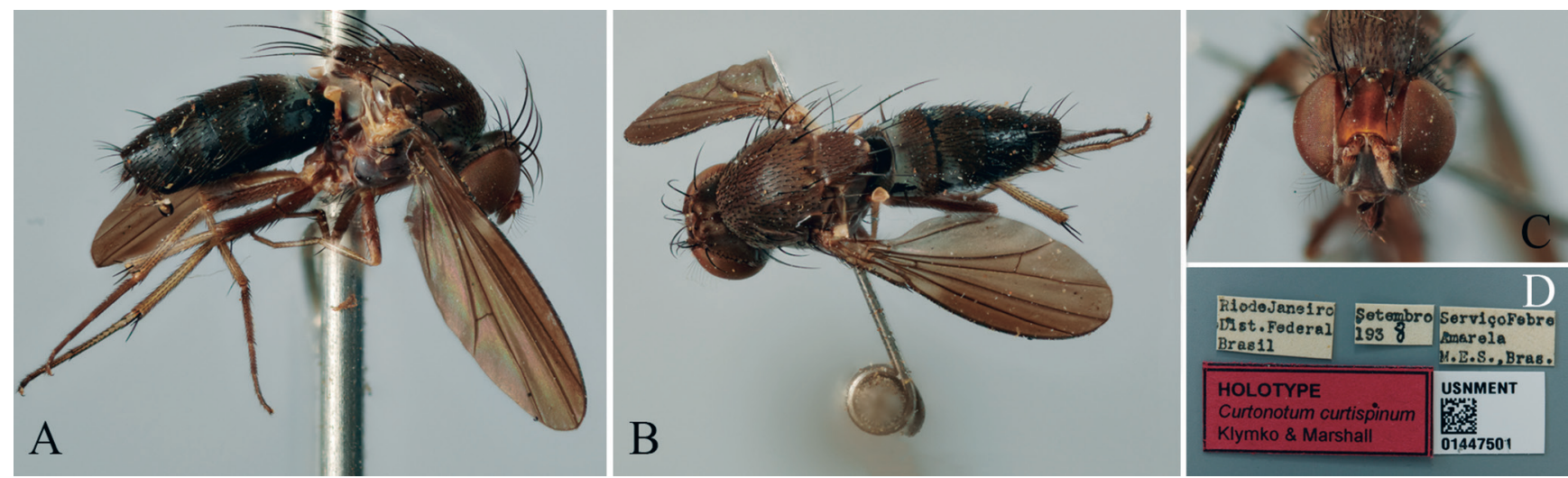

Figure 3. Curtonotum curtispinum Klymko \& Marshall, 2011, holotype male. (A) Habitus, lateral view; (B) Habitus, dorsal view; (C) Head, frontal view; (D) Labels. 
floridense Klymko \& Marshall, 2011: 27. Type-locality: USA, Florida, Polk county. Distr.: USA. Refs.: Klymko \& Marshall, 2011: 11-15 (phyl. rels.), 25 (key). Holotype male (DEBU).

fumipenne Hendel, 1913: 626. Type-locality: BOLIVIA, Mapiri, Sarampioni. Distr.: Bolivia. Refs.: Hendel, 1913: 619 (key); Sturtevant, 1921: 114 (ch.), 123 (cat.); Fischer, 1933: 88 (ch.); Wirth, 1975: 2 (cat.); Klymko \& Marshall, 2011: 64 (tax. notes). Syntypes male and female (SMTD).

gracile Klymko \& Marshall, 2011: 88. Type-locality: BRAZIL, Pará, Cachimbo ridge. Distr.: Brazil. Refs.: Klymko \& Marshall, 2011: 11-15 (phyl. rels.), 26 (key). Holotype male (MZUSP).

hendelianum (Enderlein), 1917: 72 (Diplocentra). Typelocality: SURINAME. Distr.: Suriname. Refs.: Fischer, 1933: 87 (comb.); Wirth, 1975: 2 (cat.); Klymko \& Marshall, 2011: 11-15, figs. 1-5 (phyl. rels.); 26 (key), 83 (reds.). Holotype female (HNHM).

nigripalpe Hendel, 1936: 90. Type-locality: BRAZIL, Pará, Belém. Distr.: Brazil, Guyana, Suriname. Refs.: Wirth, 1975: 2 (cat.); Papavero, 2002: 1 (cat.); Klymko \& Marshall, 2011: 11-15 (phyl. rels.), 83 (syn.). Holotype female (NHMW).

hunkingi Klymko \& Marshall, 2011: 97. Type-locality: BRAZIL, Paraná, Bocaiúva do Sul. Distr.: Brazil. Refs.: Klymko \& Marshall, 2011: 11-15 (phyl. rels.), 26 (key). Holotype male (USNM).

impunctatum Hendel, 1913: 625. Type-locality: PERU, Huanúco, Mouth of Pachieta River. Distr.: Ecuador, Peru. Refs.: Hendel, 1913: 619 (key); Sturtevant, 1921: 114 (ch.), 123 (cat.); Fischer, 1933: 88 (ch.); Wirth, 1975: 2 (cat.); Klymko \& Marshall, 2011: 11-15 (phyl. rels.), 25 (key), 58 (reds.), 61 (lect. design.). Lectotype male (SMTD).

magnum Malloch, 1930: 326 (Cyrtonotum). Type-locality: BRAZIL, Rio de Janeiro, Itatiaia, Serra do Itatiaia. Distr.: Brazil. Refs.: Wirth, 1975: 2 (cat.); Klymko \& Marshall, 2011: 11-15 (phyl. rels.), 26 (key), 45 (reds.). Holotype female (USNM).

murinum Hendel, 1913: 622. Type-locality: PERU, Chanchamayo, Meshagua, Urubamba river. Distr.: Peru. Refs.: Hendel, 1913: 619 (key); Sturtevant, 1921: 114 (ch.), 123 (cat.); Fischer, 1933: 88 (ch.); Wirth, 1975: 2 (cat.); Klymko \& Marshall, 2011: 50 (tax. notes). Syntypes male and female (SMTD).

nigrum Klymko \& Marshall, 2011: 37. Type-locality: BRAZIL, Paraná, Curitiba. Distr.: Brazil. Refs.: Klymko \& Marshall, 2011: 11-15 (phyl. rels.), 26 (key). Holotype male (MZUSP).

pantherinum (Walker), 1849: 1090 (Helomyza). Typelocality: BRAZIL. Distr.: Bolivia, Brazil, Guyana, Peru, Suriname, Venezuela. Refs.: Hendel, 1913: 619 (key), 623 (comb. reds.); Malloch, 1930: 325 (tax. notes); Fischer, 1933: 88 (ch.); Wirth, 1975: 2 (cat.); Klymko \& Marshall, 2011: 11-15, figs. 1-5 (phyl. rels.); 26 (key), 67 (reds.). Syntype male (NHM: lost, see Klymko \& Marshall, 2011: 70).

salinum Curran, 1934: 440. Type-locality: GUYANA, Kartabo. Distr.: Guyana. Refs.: Malloch, 1930: 325 (syn. of $C$. pantherinum before the formal description of the species: Klymko \& Marshall, 2011: 70). Wirth, 1975: 2 (cat.); Klymko \& Marshall, 2011: 11-15 (phyl. rels.), 70 (tax. notes). Holotype male (AMNH).

papillatum Klymko \& Marshall, 2011: 99. Type-locality: BRAZIL, Rio de Janeiro, Rio de Janeiro. Distr.: Brazil. Refs.: Klymko \& Marshall, 2011: 11-15 (phyl. rels.), 26 (key). Holotype male (USNM).

perplexum Klymko \& Marshall, 2011: 50. Type-locality: BOLIVIA, Pilcomayo. Distr.: Bolivia. Refs.: Hendel, 1932: 134 (desc. of Cyrtonotum impunctatum preoccupied by Curtonotum impunctatum Hendel, 1913); Wirth, 1975: 2 (cat.); Klymko \& Marshall, 2011: 50 (n. nam.). Holotype female (SMNS).

punctithorax Fischer, 1933: 88 (Cyrtonotum) (Fig. 1). Type-locality: BRAZIL, São Paulo, São Paulo. Distr.: Brazil, Paraguay, Uruguay. Refs.: Wirth, 1975: 2 (cat.); Klymko \& Marshall, 2011: 11-15 (phyl. rels.), 26 (key), 102 (reds.). Lectotype male (MZUSP).

Comments: Klymko \& Marshall, 2011 redescribed the species based on a male and one female cited as paratypes. In fact, these specimens are paralectotypes as the type series was designated as cotypus. Below, it is established the lectotype based on a male specimen from MZUSP. Besides the lectotype and the five paralectotypes designated below, the original type series is composed by 75 specimens (35 males and 40 females). The material indicated as hosted on author's collection (IBSP) was not found.

Studied material: Lectotype (hereby designated): male (MZUSP) "[white label] Cyrtonotum punctithorax / Fischer, 1933 | [white label] Fliegenfalle / São Paulo / 2-2-1933 | [red, black bordered label] Cotypus | [white label] ơ". Paralectotypes, hereby designated: male (NHMW) "São Paulo Capital / 14. Dez. 1932 / Carlos R. Fischer, leg. $\left|\sigma^{\prime}\right|$ Curtonotum punctithorax, Fischer. det. Wirth, 1913"; female (MZUSP) "Cyrtonotum punctithorax| Fischer, 1933 | Cotypus | Fliegenfalle / São Paulo / 31-1-1933 | O"; male (USNM) "Cotypus | São Paulo, Brasil / Capital, 30-1-1933 / C.R. Fischer leg. Cyrtonotum punctithorax / Fischer, 1933 o"; female (USNM) "Cotypus | São Paulo, Brasil / Capital, 30-1-1933 / C.R. Fischer leg. | Cyrtonotum punctithorax / Fischer, 1933 ᄋ"; female (USNM) "Cotypus | São Paulo, Brasil / Capital, 5-2-1933 / C.R. Fischer leg. | Cyrtonotum punctithorax / Fischer, 1933 \%".

scambum Klymko \& Marshall, 2011: 86. Type-locality: PERU, Loreto, Iquitos. Distr.: Peru. Refs.: Klymko \& Marshall, 2011: 11-15 (phyl. rels.), 26 (key). Holotype male (USNM).

simplex Schiner, 1868: 237 (Fig. 2) stat. rev. Type-locality: BRAZIL. Distr.: Brazil, Mexico (probably misid.). Refs.: Giglio-Tos, 1895: 36 (Diplocentra comb., cat.); Wulp, 1897: 354 (ch., in Diplocentra); Aldrich, 1905: 641 (Cyrtonotum comb., cat.); Hendel, 1913: 618 (key: as Curtonotum); Sturtevant, 1921: 114 (ch.), 124 (cat.); Wirth, 1975: 2 (cat.); Klymko \& Marshall, 2011: 107 (tax. notes). Holotype female (NHMW).

Comments: Curtonotum simplex undoubtedly belongs to the same species-group comprising C. im- 
puctatum and C. curtispinum. In many features it agrees with the proposed description for C. curtispinum, including coloring patterns, setae positioning and wing appearance. Both species have similar type localities, coming from Rio de Janeiro state, Brazil. Although we have the pictures of the type material in hand, a more detailed analysis (specimens in hands) is necessary for the taking of nomenclature acts. Probably C. curtispinum is a junior synonym of C. simplex, but they should be treated as different species until further analysis.

taeniatum Hendel, 1913: 629. Type-locality: PERU, Ucayali, Urubamba River. Distr.: Bolivia, Brazil, Peru. Refs.: Hendel, 1913: 620 (key); Enderlein, 1917: 69 (n. occ.); Sturtevant, 1921: 114 (ch.), 124 (cat.); Malloch, 1930: 326 (syn. of C. gibbum (Fabricius, 1805)); Fischer, 1933: 88 (ch.); Wirth, 1975: 2 (cat.); Thompson \& Pont, 1993: 80 (syn. and replacement name for Musca gibba Fabricius, 1805); Klymko \& Marshall, 2011: 11-15 (phyl. rels.), 25 (key), 75 (reds.), 79 (lect. desing.). Lectotype male (SMTD).

gibba (Fabricius), 1805: 297 (Musca). Type-locality: South America. Distr. Brazil, Colombia, Guyana, Mexico, Suriname. Refs.: Wiedemann, 1830: 586 (comb. in Helomyza); Macquart, 1843: 350 (comb. in Curtonotum), pl. 25 (figs. 9-10); Rondani, 1848: 78 (sync. list); Walker, 1849: 1090 (sync. list); Schiner, 1864: 23 (tax. notes); Schiner, 1868: 237 (tax. notes); Giglio-Tos, 1895: 36 (syn. in Diplocentra); Wulp, 1897: 353 (ch., in Diplocentra); Aldrich, 1905: 641 (cat.); Coquillett, 1910: 529 (tax. notes); Hendel, 1913: 619 (key, as gibbum), 625 (tax.); Enderlein, 1917: 70 (sync. list, tax. notes), 71 (n. occ.); Frey, 1919: 14 (ch.); Sturtevant, 1921: 33 (notes, as gibbum), 114 (ch.), 123 (cat.); Hendel, 1933: 77 (sync. list); Wirth, 1975: 2 (cat.); Thompson \& Pont, 1993: 80 (syn. and replacement name for Musca gibba Fabricius); Papavero, 2002: 1 (cat.); Klymko \& Marshall, 2011: 79 (lect. design.). Lectotype female (ZMUC).

trypetipenne Hendel, 1913: 620. Type-locality: PERU, Ucayali, Unini. Distr.: Brazil, Bolivia, Colombia, Ecuador, Peru. Refs.: Hendel, 1913:619 (key); Enderlein, 1917:71 (n. comb. in Diplocentra, reds.); Sturtevant, 1921: 114 (ch.), 124 (cat.); Fischer, 1933: 88 (ch.); Wirth, 1975: 2 (cat.); Klymko \& Marshall, 2011: 25 (key), 55 (reds.), 57 (lect. design.); Mello, 2016: 717 (cat.). Lectotype female (SMTD).

tumidum Enderlein, 1917: 68 (Cyrtonotum). Typelocality: SURINAME. Distr.: Brazil, Colombia, Ecuador, Guyana, Peru, Suriname. Refs.: Fischer, 1933: 88 (ch.); Wirth, 1975: 3 (cat.); Papavero, 2002: 1 (cat.); Klymko \& Marshall, 2011: 11-15 (phyl. rels.), 26 (key), 80 (reds.), 83 (lect. design.); Mello, 2016: 717 (cat.). Lectotype male (HNHM).

vulpinum Hendel, 1913: 627. Type-locality: PERU, Pichis, Puerto Bermudez. Distr.: Bolivia, Peru. Refs.: Hendel, 1913: 619 (key); Enderlein, 1917: 69 (n. occ.); Sturtevant, 1921: 114 (ch.), 124 (cat.); Fischer, 1933: 88 (ch.); Wirth, 1975: 3 (cat.); Klymko \& Marshall, 2011: 11-15 (phyl. rels.), 25 (key), 64 (tax. notes). Syntypes male and female (SMTD).

\section{Depressonotum Grimaldi \& Kirk-Spriggs, 2012}

Depressonotum Grimaldi \& Kirk-Spriggs, 2012: 10. Type species: Depressonotum priscum Grimaldi \& KirkSpriggs, 2012 (orig. des.).

tpriscum Grimaldi \& Kirk-Spriggs, 2012: 10. Type-locality: DOMINICAN REPUBLIC, amber from Cordillera Septentrional, northern Dominican Republic. Distr.: Dominican Republic (Miocene). Holotype male (AMNH).

\section{Nomen dubium according to Klymko \& Marshall, 2011}

hendeli Malloch, 1930: 325 (Cyrtonotum). Type-locality: BRAZIL. Distr.: Brazil, Guyana. Refs.: Hendel, 1913: 624 (Curtonotum gibbum: misidentification); Malloch, 1930: 325 (new name and combination to Curtonotum gibbum sensu Hendel, 1913); Curran, 1934: 441 (reds., n. occ.); Wirth, 1975: 2 (cat.); Klymko \& Marshall, 2011: 106 (tax. notes). Syntypes (USNM).

\section{ACKNOWLEDGMENTS}

The authors would like to thank Mrs. Camila Conti (MZUSP) for the photos of the lectotype of C. punctithorax and to the MSc. Sheila Lima (INPA) for the remarks about the type locality of $C$. flavisetum. We are indebted to Sergio Ide (Instituto Biológico, SP), Torsten Dikow (USNM) by the photos of the holotype of C. curtispinum (USNMENT: 01447501) and Peter Sehnal (NHMW) for the information about $C$. punctithorax type series and for the photos of $C$. simplex. We would like to thank Soltész Zoltán (HNHM) for confirming the presence of the types in his collection. APC is grateful to Fundação de Apoio à Pesquisa do Estado da Paraíba (FAPESQ) and Conselho Nacional de Desenvolvimento Científico e Tecnológico (CNPq) for the research support (Proc. \#350052/2014-0).

\section{REFERENCES}

Agassiz, L. 1846. Nomenclatoriszoologici index universalis, continens nomina systematica classium, ordinum, familiarum et generum animalium omnium, tam viventium quam fossilium, secundum ordinem alphabeticumu nicum disposita, adjectis homonymiis plantarum, nec non variis adnotationibus et emendationibus. Sumptibus et typis Jent et Gassmann, Soloduri (= Solothurn, Switzerland), 393 pp. D0l

Aldrich, J.M. 1905. A catalogue of North American Diptera. Washington, Smithsonian Institution. 680p. DOI

Bezzi, M. 1895. Contribuzioni alla fauna ditterologica italiana. Bolletino della Societá entomologica italiana, 27: 39-78. https://biodiversitylibrary.org/ page/10912858.

Bezzi, M. 1914. Eine neue südamerikanische Art der Dipteran gattung Cyrtonotum. Deutsche Entomologische Zeitschrift, 7: 199-200. https:// biodiversitylibrary.org/page/33061920.

Coquillett, D.W. 1910. The type-species of the North America genera of Diptera. Proceedings of the United States National Museum, 37: 499-647. D01 
Curran, C.H. 1934. The Diptera of Kartabo, Bartica District, British Guiana, with descriptions of new species from other British Guiana localities. Bulletin of the American Museum of Natural History, 66: 287-532. http:// digitallibrary.amnh.org/handle/2246/1248.

Delfinado, M.D. 1969. The Oriental species of Curtonotidae (Diptera). Oriental Insects, 3: 199-219. DOI

Duda, 0. 1924. Beitrag zur Systematik der Drosophiliden unter besonderer Berücksichtigung der Palaarktischen und Orientalischen Arten (Dipteren). Archiv für Naturgeschichte, 90: 172-234. D01

Duda, 0.1934.Curtonotidae.In:Lindner, E. (Ed.).DieFliegenderPalaearktischen Region. Stuttgart, Schweizerbartsche Verlags-buchhandlung. v. 6, pt. 1, p. 1-5.

Enderlein, G. 1914. Ordnung Diptera, Fliegen (Zweiflugler). In: Brohmer, P. (Ed.). Fauna von Deutschland. Leipzig, Verlag von Quelle und Meyer. p. 272-334.

Enderlein, G. 1917. Dipterologische Studien. XVI. Dipterologische Notizen. Zoologischer Anzeiger, 49: 65-72. https://www.biodiversitylibrary.org/ part/68698.

Fabricius, J.C. 1805. Systema Rhyngotorum secundum ordines, genera, species adiectis synonymis, locis, observationibus, descriptionibus. Brunsvigae, C. Reichard. 403p. DOI

Fischer, C.R. 1933. Nota sobre Anastrepha punctata Hend. (Dipt. Trypetidae) e uma espécie nova de Cyrtonotum (Dipt. Drosophilidae). Revista de Entomologia, 3: 83-92.

Frey, R. 1919. Mitteilungen über südamerikanische Dipteren. Öfversigt af Finska vetenskaps-societetens förhandlingar, 60(14): 1-35. https:// biodiversitylibrary.org/page/14804176.

Giglio-Tos, E. 1895. Ditteridel Messico. Parte IV. Muscidae CalypterataeMuscidaeAcalypteratae. Torino, C. Clausen. 73p. https:// biodiversitylibrary.org/page/8616389.

Greathead, D.J. 1958. Notes on the larva and life history of Cyrtonotum cuthbertsoni Duda (Dipt., Drosophilidae), a fly associated with the desert locust Schistocerca gregaria (Forskål). Entomologist's Monthly Magazine, 94: 36-37.

Grimaldi, D.A. \& Kirk-Spriggs, A.H. 2012. Fossil Curtonotidae (Diptera: Schizophora: Ephydroidea). American Museum Novitates, 3760: 1-16. http://hdl.handle.net/2246/6392.

Hendel, F. 1913. Neue amerikanische Diptera. 1. Beitrag. Deutsche Entomologische Zeitschrift, 1913: 617-636. https://biodiversitylibrary. org/page/33050094.

Hendel, F. 1932. Die Ausbeute der deutschen Chaco-Expedition 1925/26. (Fortsetzung.) - Diptera. XXX.-XXXVI. Sciomyzidae, Lauxaniidae, Tanypezidae, Lonchaeidae, Tylidae, Drosophilidae, Milichiidae(Schluß.). Konowia, 11: 115-145. https://www.zobodat.at/publikation articles. php?id=59437.

Hendel, F. 1933. Ueber einige Typen Wiedemann's und Schiner's von acalypraten Musciden aus Südamerika, nebst einigen verwandten Arten (Dipt.). Revista de Entomologia, 3: 58-83.

Hendel, F. 1936. Ergebnisse einer zoologischen Sammelreise nach Brasilien, insbesondere in das Amazonasgebiet, ausgeführt von Dr. H. Zerny. X. Teil. Diptera: Muscidae Acalyptratae (excl. Chloropidae). Annalen des Naturhistorischen Museums in Wien, 47: 61-106. http://www.zobodat. at/publikation articles.php?id=78145.

Hennig, W. 1958. Die Familien der Diptera Schizophora und ihre phylogenetischen Verwandtschaftbeziehungen. Beiträge zur Entomologie, 8: 505-688. https://www.contributions-to-entomology. org/article/view/398/397.

ICZN (International Commission on Zoological Nomenclature). 1999. International Code of Zoological Nomenclature. Fourth edition, adopted by the International Union of Biological Sciences. London, International
Trust for Zoological Nomenclature, The Natural History Museum. $\mathrm{xxx}+306 \mathrm{p}$.

Kirk-Spriggs, A.H. 2008. A contribution to the knowledge of the immature stages of Curtonotum (Diptera: Curtonotidae), from Africa and the Middle East, with a discussion of relationships to other known Ephydroidea larvae. African Entomology, 16(2): 226-243. D01

Kirk-Spriggs, A.H. 2010a. A revision of Afrotropical Quasimodo flies (Diptera: Curtonotidae: Schizophora). Part I - the genus Axinota van der Wulp, with the description of three new species. African Entomology, 18(1): 99-126. DOI

Kirk-Spriggs, A.H. 2010b. A revision of Afrotropical Quasimodo flies (Diptera: Curtonotidae: Schizophora). Part II - the East African Afromontane genus Tigrisomyia gen. n., with descriptions of four new species. African Entomology, 18(1): 127-146. D0I

Kirk-Spriggs, A.H. 2011. A revision of Afrotropical Quasimodo flies (Diptera: Schizophora; (urtonotidae). Part III - the Malagasy species of Curtonotum Macquart, with descriptions of six new species. African Invertebrates, 52(2): 391-456.

Kirk-Spriggs, A.H. \& Wiegmann, B.M. 2013. A revision of Afrotropical Quasimodo flies (Diptera: Schizophora; Curtonotidae). Part IV the continental Afrotropical species of Curtonotum Macquart, with descriptions of thirteen new species and a combined phylogenetic analysis of the Curtonotidae. Zootaxa, 3684(1): 1-166. https://10.11646/ zootaxa.3684.1.1.

Klymko, J. \& Marshall, S.A. 2011. Systematics of New World Curtonotum Macquart (Diptera: Curtonotidae). Zootaxa, 3079: 1-110.

Loew, H. 1862. Ueber die europäischen Helomyzidae und die in Schlesien vorkommenden Arten derselben. Zeitschrift für Entomologie, 13: 1-80. http://publikationen.ub.uni-frankfurt.de/frontdoor/index/index/ docld/9682.

Lucas, R. 1915. Diptera für 1914: Systematik (part). Archiv für Naturgeschichte, 8(B): 81-193.

Macquart, P.-J.-M. 1843. Diptères exotiques nouveaux ou peu conns. Mémoires de la Société royale des sciences, de l'agriculture et des arts de Lille, 1842: 162-460. https://www.biodiversitylibrary.org/item/54217\#page/7/ mode/1up.

Malloch, J.R. 1930. Exotic Muscaridae (Diptera). - XXX. Annals and Magazine of Natural History, (10)6: 321-334.

Marshall, S.A. 2012. Flies: The Natural History and Diversity of Diptera. New York, Firefly Books. 616p.

Marshall, S.A.; Kirk-Spriggs, A.H. \& Klymko, J. 2010. Curtonotidae. In: Brown, B.V.; Borkent, A.; Cumming, J.M.; Wood, D.M.; Woodley, N.E. \& Zumbado, M. (Eds.). Manual of Central American Diptera. Ottawa, National Research Council Press. v. 2, p. 21189-1192.

Meier, R.; Kotrba, M. \& Barber, K. 1997. A comparative study of the egg, firstinstar larva, puparium, female reproductive system and natural history of Curtonotum helvum (Curtonotidae; Ephydroidea; Diptera). American Museum Novitates, 3219: 1-20. http://hdl.handle.net/2246/3617.

Mello, R.L. 2016. Family Curtonotidae. Zootaxa, 4122: 716-718. https:// biotaxa.org/Zootaxa/article/view/zootaxa.4122.1.62.

Papavero, N. 2002. Insecta, Diptera, Curtonotidae. Fauna da Amazônia Brasileira, Belém, 7: 1-2.

Rondani, C. 1848. Esame di varie specie di insetti ditteri brasiliana. Studi Entomologici, 1: 63-112. https://www.biodiversitylibrary.org/ page/40161207\#page/71/mode/1up.

Schiner, I.R. 1864. Fauna Austriaca: die Fliegen (Diptera) Nach der analytischen Methode bearbeitet, mit der Characteristik sälmmtlicher europäischer Gattungen, der Beschreibung aller in Deutschland vorkommenden Arten und der Aufzählung aller bisher beschriebenen europäischen Arten. Wien, Druck und Verlag von Carl 
Gerold's Sohn. xxxii + 658p. https://www.biodiversitylibrary.org/ item/37727\#page/65/mode/1up.

Schiner, I.R. 1868. Diptera. In: Reise der Österreichischen Fregatte Novara um die Erde in den Jahren 1857, 1858, 1859, unter den Befehlen des Commodore B. Von Wüllerstorf-Urbair. Wien, Kaiserlich-Koniglischen Hof-und Staatsdrukerel Zoologischer Theil 2 I(B), p. 237. https://www. biodiversitylibrary.org/item/16569\#page/251/mode/1up.

Sturtevant, A.H. 1921. The North American species of Drosophila. Lancaster, Pennsylvania, New Era Publishing Co. 150p. (Publ. 301, Carnegie Institution of Washington). https://www.biodiversitylibrary.org/ item/41372\#page/7/mode/lup.

Thompson, F.C. \& Pont, A.C. 1993. Systematic database of Musca names (Diptera). A catalog of names associated with the genus-group name Musca Linnaeus, with information on their classification, distribution, and documentation. ThesesZoologicae, 20: 1-221. https://repository. si.edu/handle/10088/18919.
Walker, F. 1849. List of the specimens of dipterous insects in the collection of the British Museum Part IV. London, Printed by the order of the Trustees. p. 689-1172. https://www.biodiversitylibrary.org/ item/118982\#page/412/mode/1up.

Wiedemann, C.R.W. 1830. Aussereuropäische zweifluegeliche Insekten 2. Hamm Schulzischen Buchhandlung. xii. 684p. https://www. biodiversitylibrary.org/item/88495\#page/604/mode/1up.

Wirth, W.W. 1975. Family Curtonotidae. In: Papavero, N. (Ed.). A catalogue of the Diptera of the America South of the United States. São Paulo, Departamento de Zoologia, Secretaria de Agricultura. n. 78, 5p. https:// biodiversitylibrary.org/page/50661013.

Wulp, F.M. van der. 1897. Muscidae Acalypterae (cont.). In: Godman, F.D. \& Salvin, 0. (Eds.). Biologia Centrali-Americana or, contributions to the knowledge of the fauna and flora of Mexico and Central America. Zoologia. Class Insecta. Order Diptera, London, Taylor \& Francis, v. 2, p. 353-361. https://www.biodiversitylibrary.org/item/14628\#page/365/mode/1up. 